\title{
AN UNSUPERVISED OUTLIER DETECTION METHOD FOR 3D POINT CLOUD DATA
}

\author{
Emon Kumar Dey, Mohammad Awrangjeb, Bela Stantic \\ School of Information and Communication Technology, Griffith University \\ Nathan, QLD 4111, Australia
}

\begin{abstract}
This paper introduces an effective method for outlier detection from the point cloud data. Although, the state-of-the-art methods offer good results in removing outliers, in most of the cases inliers are also removed erroneously. This paper focuses on this issue using the information based on a relative location from a point to its neighbours and a robust $z$-score based on a statistical approach. Synthetic datasets for 3D building roofs have been created to evaluate the performance. When compared with the existing methods, the proposed method exhibits better performance, i.e., $19 \%$ more recall for inliers, $6 \%$ more precision for outliers and $10 \%$ more overall accuracy. In other words, it not only preserves the inliers, but also correctly removes the outliers with a better precision rate than the current state-of-the-arts methods.
\end{abstract}

Index Terms - Outlier Detection, Point Cloud, Local Outlier, Point Cloud Denoising

\section{INTRODUCTION}

Presence of outliers in point cloud data is inexorable. Limitations or unexpected behaviour of the data collector sensors, multiple reflections, natural variations etc. are the common causes of outliers in point cloud data. Dataset impinged with different types of outliers degrades the performance of different applications. For example, using LiDAR (Light Detection and Ranging) point cloud data building roof extraction [1], $3 \mathrm{D}$ reconstruction of buildings in a city [2] and solar potential estimation [3] can be affected by presence of outliers. A set of outliers may create false building planes which leads to a serious fault for the automated system. Removal of outliers is an inevitable pre-processing in these cases.

Point cloud data are usually unorganised, inconsistent with respect to densities and also geometrically inharmonious. These phenomena make the outlier detection process complex and difficult. It is a process of identifying the data which do not show the expected behaviours and can be separated from the pattern of the majority. Outliers need to be removed but it also should be in consideration that the inliers must be retained. In some applications, incorrectly removed inliers create missing data problem which is more harmful. So, a method which can detect and remove the outliers and at the same time keep the inliers as complete as possible will be more effective.

Both supervised and unsupervised approaches exists to detect the outliers from point cloud data. Supervised approaches are effective for many classification tasks. But in case of outlier detection, this approach is complex in reality for various reasons. First of all, the accurate labelled data with inliers and outliers are not easily available to train the system. On the other hand, outliers exhibits dynamic natures and properties. So, suitable features are difficult to figure out the nature of outliers.

Chandola et al. [4] classified three types of outliers: point, collective and contextual outliers. A point outlier shows significant difference from the rest of the dataset. In collective outlier, a group of data shows the deviation while contextual outliers are the instances which are different in a specific context. Point outliers is subdivided into local and global outliers. If the global outliers are sparsely distributed in point cloud data then a method designed for detecting local outlier can also detect the global outliers [5].

In this paper, we propose an unsupervised outlier detection method contemplating to make the roof extraction or 3D modelling more robust. The statistical approach, which is mainly based on distance measures from the neighbourhood of a 3D point is used for the method. We create synthetic datasets with inliers and outliers and compare the result of the proposed method with the two state-of-the-art methods $[6,7]$. Section 2 describes the related works and the proposed method is presented in Section 3. Experimental results with the test datasets are demonstrated in Section 4, and finally, Section 5 concludes the paper.

\section{RELATED WORK}

Outlier detection methods from point cloud data have been developed and implemented both in supervised and unsupervised classification approaches in various area including machine learning, pattern recognition, hyperspectral image processing, statistics and data mining.

In supervised approach, a model is trained using some known labelled data. The model uses a classifier for the training purpose. The test data points are classified as either inliers or outliers based on the decision of the classifier. Liu et 
al. [8] proposed an Support Vector Data Descriptor (SVDD) based approach to detect outliers on uncertain data which operate on two steps. In the first step, they generated a pseudo training set using a confident score for each sample and, in the second step, the set was used for training a global SVDD classifier. Recently Stucker et al. [5] used standard Random Forest Classifier to find out the label (inlier or outlier) of each input 3D points. They applied semantic and nonsemantic approaches in their paper. In the semantic approach they trained the classifier incorporating inliers and outliers for each semantic class like roof, vegetation, ground, water etc. In the non-semantic approach, features extraction and classifier training were performed without considering the interpretations of individual classes.

Unsupervised approach for outlier detection is the most common because of unknown behaviours of outliers and unavailability of labelled ground truth data. These methods mainly follow statistical formula, distance and density based techniques. In statistical techniques the outliers are identified based on a standard probability distribution [9]. Rusu and Cousins [10] assumed that the inlier points show a Gaussian distribution and the statistical hypothesis testing of outliers exhibits more than a certain confidence interval. Nurunnabi et al. [7] proposed two methods for outlier detection using robust statistical techniques. The first method is based on a robust $z$-score and the second method used the Mahalanobis distance to identify the outliers. Zang et al. [6] calculated the outlier-ness of a point in scattered data by proposing the Local Distance-based Outlier Factors (LDOF). This method identified the relative location of the point to its neighbour and found the degree to which the point deviated from its neighbours. According to Luo et al. [11] LDOF performs better in 2D sliced and projected points, while 3D neighbours of a point in LDOF degrade its performance. Clustering based unsupervised approaches has also been applied to remove outliers [12]. Awrangjeb et al. [13] removed the small clusters which were less than $1 \mathrm{~m}^{2}$. They used the term 'false plane' instead of outlier in their paper. The false planes contained significantly less data points than the true planes.The performance of their approach highly depended on the clustering algorithm applied on the input data.

The proposed unsupervised method is based on a statistical analysis and relative distances among the neighbouring points in point cloud. Delaunay triangulation has been used for calculating the natural neighbourhood.

\section{PROPOSED METHOD}

As mentioned earlier, outlier can be divided into two categories: local outliers and global outliers. Finding the global outliers is not a congenial way because global outliers are sparse and do not show distinctive geometric attributes. Sometimes global outliers form micro clusters which mislead the detection process. The method proposed in this paper tries to find out the local outliers using the information of the neighbours of each 3D point. We mainly adopt the methods Maximum Consistency with Minimum Distance (MCMD) using Robust $z$-score $\left(R_{z}\right)$ (see eq.(1)) and LDOF (see eq.(2)) proposed in Nurunnabi et al. [7] and Zang et al. [6], respectively. Both of these methods used local neighbouring points to make their decision about outliers and applied the $k$-Nearest Neighbourhood algorithm $(k-\mathrm{NN}) . R_{z}$ score only gives an indication of relative Orthogonal Distance (OD) from a point to the plane created by the neighbouring points and LDOF provides the relative location of a point to its neighbours. Our proposed method considers both of the scores for making the decision about an inlier. Since some inliers may be misclassified by considering only $R_{z}$ score [7] or the relative distance alone in 3D point cloud [6], the relative distance and $R_{z}$ score together give a better decision for inliers.

Let $N_{p}=\left\{q_{j}\right\}$ where $j \geq 1$ be the set of all neighbours of a point $p_{i}$ in the point cloud data, $n$ be the cardinality of $N_{p}, d_{p_{i}}$ be the average distance from all neighbours in $N_{p}$ to $p_{i}$ and $D_{p_{i}}$ be the average distance among all points in $N_{p}$ and $|.$.$| indicate the absolute value.$

$$
\begin{gathered}
R_{z_{i}}=\frac{\left|p_{i}-\operatorname{median}\left(N_{p}\right)\right|}{\underset{i}{\text { a.median }\left|p_{i}-\operatorname{median}\left(N_{p}\right)\right|}}, i=1 \ldots . . . \\
\operatorname{LDOF}\left(p_{i}\right)=\frac{d_{p_{i}}}{D_{p_{i}}}
\end{gathered}
$$

where

$$
d_{p_{i}}=\frac{1}{n} \sum_{q_{j} \in N_{p}} \operatorname{dist}\left(q_{j}, p_{i}\right)
$$

and

$$
D_{p_{i}}=\frac{1}{n(n-1)} \sum_{q_{j}, q_{j^{\prime}} \in N_{p}, j \neq j^{\prime}} \operatorname{dist}\left(q_{j}, q_{j^{\prime}}\right)
$$

Our proposed algorithm first calculates $R_{z}$ score of a specific point using MCMD_Z algorithm. We choose $a=1.483$ in eq.(1) as a correction factor to make the $R_{z_{i}}$ score consistent [9]. Next, the algorithm finds the LDOF score for each of the 3D points in a cluster. If a point exceeds the predefined thresholds $T_{z}$ and $T_{l}$ for $R_{z}$ and LDOF, respectively, then we consider the point as an outlier. The proposed outlier detection method is summarised in Algorithm 1.

Although the well-known neighbourhood searching methods $k$-NN [7] and different forms of Fixed Distance Neighbourhood (FDN) [14] have been used by many researchers for point cloud analysis, we use the delaunay triangulation to calculate neighbourhood as it gives the natural neighbouring points and free from choosing the values like $k$ or distance thresholds. Using our dataset, we also found that for higher $k$ values (more than 100) the existing methods (LDOF, MCMD_Z and MCMD_MD) show good results, whereas delaunay algorithm chooses approximately 10 to 15 times less 


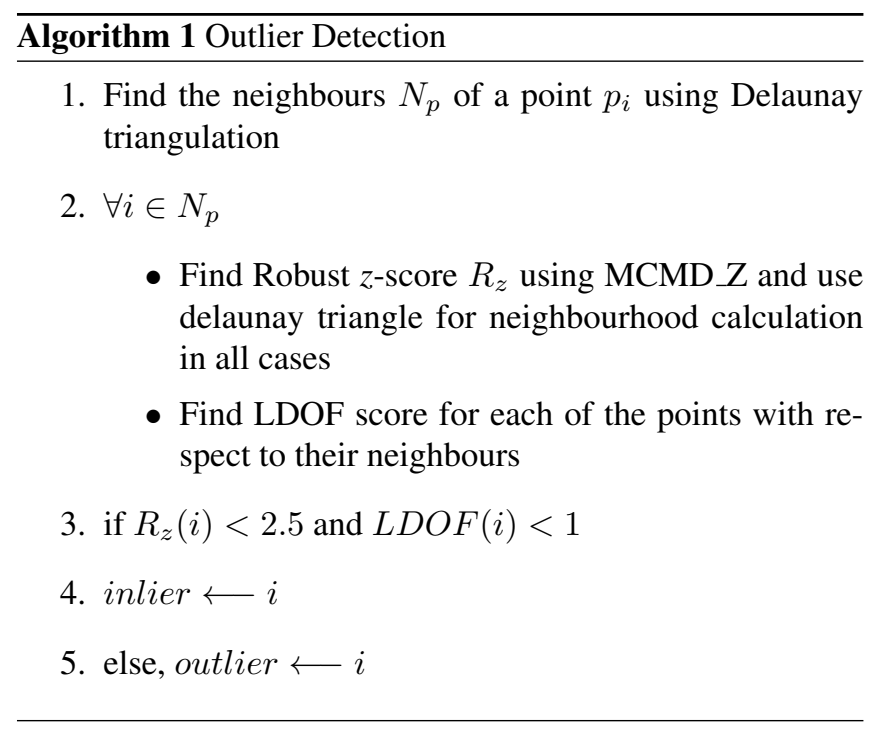

points to make the decision. This makes the proposed algorithm faster along with better results.

\section{EXPERIMENTAL RESULTS}

Major problem for outlier detection from point cloud research is the limitation of the ground truth dataset. For experiment, therefore, we applied our proposed method on two synthetic datasets we have created. The datasets and results are described in the following subsections.

\subsection{Dataset}

Available datasets are not labelled with inliers and outliers. To generate a labelled dataset we take help from the method proposed by Awrangjeb et al. [2]. The method is a data driven approach for building roof reconstruction and change detection. We first constructed a 3D building roof using their method from the LiDAR data and then created synthetic inliers and outliers on each of the roof planes.

For experimental purpose we first applied our method on a single building (SB) created from the Aitkenvale (AV) dataset. After that we applied the proposed method on the main dataset consisting of 5 different buildings. The area of the site is $66 \mathrm{~m} \times 52 \mathrm{~m}$ with point density 40 points $/ \mathrm{m}^{2}$. For the first case we manually created a total of $13603 \mathrm{D}$ points among which 1225 are inliers and 135 are outliers. Inlier points are created with orthogonal distance between 0 to \pm 0.05 meter of the roof plains. Outliers are also randomly generated for each plane with orthogonal distance from \pm 0.06 to $\pm 0.45 \mathrm{~m}$. Again we created ground truth with 3924 inliers and 612 outliers (approximately 15\%) for 5 buildings with 22 roof planes on Aitkenvale dataset.

Fig. 1 (a) shows the created inliers and outliers on a building with 8 roof planes. Red star $\left(^{*}\right)$ indicates the outliers and
Table 1. Accuracy measures using Single Building (SB) data model

\begin{tabular}{lccccc}
\hline Methods & TPR & TNR & FPR & FNR & Acc \\
\hline LDOF & 0.31 & 0.70 & 0.31 & 0.69 & 0.71 \\
MCMD_Z & 0.70 & 0.82 & 0.17 & 0.28 & 0.89 \\
MCMD_MD & 0.60 & 0.83 & 0.16 & 0.40 & 0.81 \\
Proposed & $\mathbf{0 . 7 5}$ & $\mathbf{0 . 9 7}$ & $\mathbf{0 . 0 3}$ & $\mathbf{0 . 2 4}$ & $\mathbf{0 . 9 5}$ \\
\hline
\end{tabular}

black dot (.) indicates the created inliers. Fig. 1 (b) shows the 3D models of the buildings we have used for our experiment.

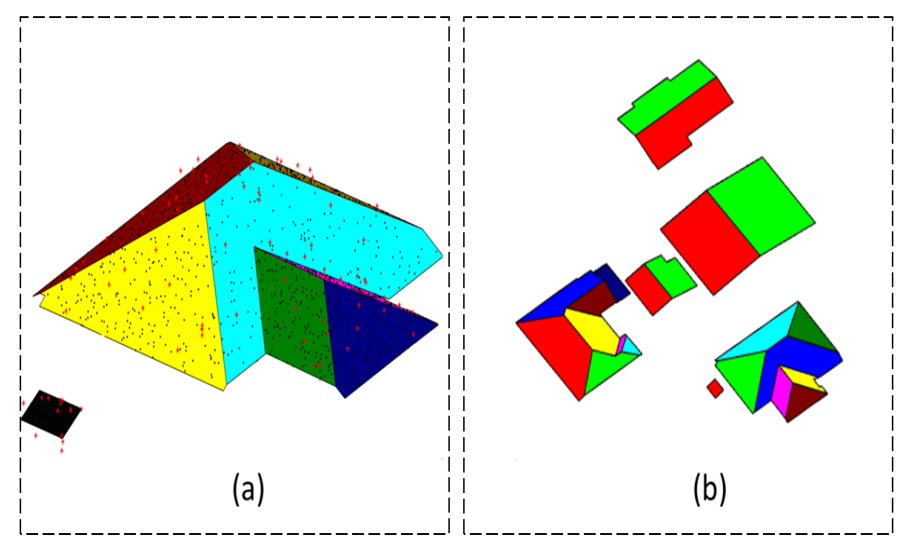

Fig. 1. Aitkenvale (AV) dataset: (a) Inliers and outliers on single building roof and (b) 3D roof models of $\mathrm{AV}$ dataset

\subsection{Results}

We find the inliers and outliers using our method and compute precision, recall, $F_{1}$-score. True Positive Rate (TPR), True Negative Rate (TNR), False Positive Rate (FPR), False Negative Rate (FNR) are also calculated using the conventional formulas. The proposed method is compared with three other methods LDOF, MCMDZZ and MCMD_MD as mentioned earlier. The ratio of the number of outliers correctly detected to the total number of outliers is defined as TPR whereas ratio of the correctly identified inliers to the total number of inliers is described as TNR. The number of inliers identified as outliers with respect to the total number of inliers is defined as FPR. Again, the ratio of the total number of outliers detected as inliers to the total number of outliers is identified as FNR.

From Table 1 and Table 2 it is clear that the proposed method performed better than the other three methods. TNR and accuracy are quite higher for both of the datasets which is the main goal of this research. Table 3 shows comparisons using Precision, Recall and $F_{1}$-score for both datasets we have used where better results by the proposed method are evident. Although we can see that our method shows a little bit lower 
Table 2. Accuracy measures using Aitkenvale (AV) five building data models

\begin{tabular}{cccccc}
\hline Methods & TPR & TNR & FPR & FNR & Acc \\
\hline LDOF & 0.53 & 0.54 & 0.49 & 0.48 & 0.54 \\
MCMD_Z & $\mathbf{0 . 8 1}$ & 0.79 & 0.18 & $\mathbf{0 . 1 9}$ & 0.86 \\
MCMD_MD & 0.72 & 0.73 & 0.26 & 0.27 & 0.76 \\
Proposed & 0.79 & $\mathbf{0 . 9 8}$ & $\mathbf{0 . 0 2}$ & $\mathbf{0 . 1 9}$ & $\mathbf{0 . 9 6}$ \\
\hline
\end{tabular}

Table 3. Comparison with different methods M1 = LDOF [6], $\mathrm{M} 2=\underline{\mathrm{MCMD} Z \mathrm{Z} \text { [7], M3 = MCMD_MD [7] }}$

\begin{tabular}{|c|c|c|c|c|c|}
\hline \multicolumn{6}{|c|}{ SB Dataset } \\
\hline \multirow{4}{*}{ 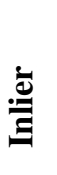 } & & M1 & M2 & M3 & Proposed \\
\hline & Precision & 0.85 & 0.97 & 0.95 & 0.97 \\
\hline & Recall & 0.75 & 0.80 & 0.83 & 0.96 \\
\hline & $F_{1}$-score & 0.79 & 0.88 & 0.89 & 0.97 \\
\hline \multirow{3}{*}{ (ֶ) } & Precision & 0.15 & 0.69 & 0.30 & 0.75 \\
\hline & Recall & 0.31 & 0.70 & 0.60 & 0.74 \\
\hline & $F_{1}$-score & 0.21 & 0.69 & 0.39 & 0.75 \\
\hline
\end{tabular}

\begin{tabular}{|c|c|c|c|c|c|}
\hline \multicolumn{6}{|c|}{ AV Dataset } \\
\hline \multirow{4}{*}{ : } & & M1 & M2 & M3 & Proposed \\
\hline & Precision & 0.87 & 0.99 & 0.95 & 0.97 \\
\hline & Recall & 0.53 & 0.72 & 0.73 & 0.98 \\
\hline & $F_{1}$-score & 0.66 & 0.82 & 0.83 & 0.97 \\
\hline \multirow{3}{*}{ 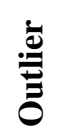 } & Precision & 0.20 & 0.60 & 0.26 & 0.84 \\
\hline & Recall & 0.52 & 0.90 & 0.71 & 0.82 \\
\hline & $F_{1}$-score & 0.28 & 0.71 & 0.39 & 0.83 \\
\hline
\end{tabular}

precision for inliers and recall for outliers than MCMD_Z for the AV dataset, it means the proposed method emphasis on inliers and misclassifies some outliers, but the overall $F_{1}$-score is quite higher than the three state-of-the-art methods.

\section{CONCLUSION}

In this paper, we have proposed an outlier detection method from the 3D point cloud data and the experimental results show better performance for inliers and outliers identification than the state-of-the-art. Most of the existing methods remove inlier points by wrongly detecting those as outliers. This is a major problem for $3 \mathrm{D}$ reconstruction because of missing true inlier points. The proposed method is almost $98 \%$ accurate for inliers and shows overall $96 \%$ accuracy. In future, we will investigate how to apply the method for 3D building reconstruction and accurate solar potential estimation of a particular area, based on point cloud data with better accuracy.

\section{REFERENCES}

[1] S.A.N Gilani, M. Awrangjeb, and G. Lu, "Segmentation of airborne point cloud data for automatic building roof extraction," GIScience \& Remote Sensing, vol. 55, no. 1, pp. 63-89, 2018.

[2] M. Awrangjeb, S. A. N. Gilani, and F. Siddiqui, "An effective data-driven method for 3-d building roof reconstruction and robust change detection," Remote Sensing, vol. 10, no. 10, 2018.

[3] D. Assouline, N. Mohajeri, and J. Scartezzini, "Largescale rooftop solar photovoltaic technical potential estimation using random forests," Applied Energy, vol. 217, pp. 189-211, 2018.

[4] V. Chandola, A. Banerjee, and V. Kumar, "Anomaly detection: A survey," ACM computing surveys (CSUR), vol. 41, no. 3, pp. 15, 2009.

[5] C. Stucker, A. Richard, J. D Wegner, and K. Schindler, "Supervised outlier detection in large-scale mvs point clouds for 3d city modeling applications.," ISPRS Annals of Photogrammetry, Remote Sensing \& Spatial Information Sciences, vol. 4, no. 2, 2018.

[6] Ke Zhang and H. Hutter, M.and Jin, "A new local distance-based outlier detection approach for scattered real-world data," in Pacific-Asia Conf. on Knowledge Disco. and Data Mining. Springer, 2009, pp. 813-822.

[7] A. Nurunnabi, G. West, and D. Belton, "Outlier detection and robust normal-curvature estimation in mobile laser scanning 3d point cloud data," Pattern Recognition, vol. 48, no. 4, pp. 1404-1419, 2015.

[8] B. Liu, Y. Xiao, L. Cao, Z. Hao, and F. Deng, "Svddbased outlier detection on uncertain data," Knowledge and information sys., vol. 34, no. 3, pp. 597-618, 2013.

[9] P. J Rousseeuw and M. Hubert, "Robust statistics for outlier detection," Wiley Interdisciplinary Reviews: Data Mining and Knowledge Discovery, vol. 1, no. 1, pp. 73-79, 2011.

[10] R. B. Rusu and S. Cousins, " $3 \mathrm{~d}$ is here: Point cloud library (pcl)," in Robotics and automation (ICRA), 2011 IEEE Int. Conference on. IEEE, 2011, pp. 1-4.

[11] D. Luo and L. Liao, "Mining outliers from point cloud by data slice," in Artificial Intelligence and Education (ICAIE), 2010 Int. Conf. on. IEEE, 2010, pp. 663-666.

[12] S. Jiang and Q. An, "Clustering-based outlier detection method," in Fuzzy Systems and Knowledge Discovery, 2008. FSKD'08. Fifth International Conference on. IEEE, 2008, vol. 2, pp. 429-433.

[13] M. Awrangjeb and C. S Fraser, "Automatic segmentation of raw lidar data for extraction of building roofs," Remote Sensing, vol. 6, no. 5, pp. 3716-3751, 2014.

[14] Soheil Sotoodeh, "Outlier detection in laser scanner point clouds," International Archives of Photogrammetry, Remote Sensing and Spatial Information Sciences, vol. 36, no. 5, pp. 297-302, 2006. 